\title{
Nicotine-induced Acute Hyperactivity Is Mediated by Dopaminergic System in a Sexually Dimorphic Manner
}

Yunpeng Zhang ${ }^{1,2}$, Jing Guo ${ }^{1}$, Aike Guo ${ }^{1,3 *}$, Yan $\mathrm{Li}^{1 *}$

${ }^{1}$ State Key Laboratory of Brain and Cognitive Science, Institute of Biophysics, Chinese Academy of Sciences, Beijing 100101, China

${ }^{2}$ University of Chinese Academy of Sciences, Beijing 100049, China

${ }^{3}$ Institute of Neuroscience, State Key Laboratory of Neuroscience, Shanghai Institutes for Biological Sciences, Shanghai 200031, China

* These authors co-supervised this research

To whom correspondence should be addressed. E-mail: liyan@ibp.ac.cn and akguo@ion.ac.cn.

Abbreviations: cAMP, cyclic adenosine monophosphate; CREB, cAMP-response element binding; CS, Canton-S; DopR, D1-like DA receptor; EB, ellipsoid body; FB, fan-shaped body; GRASP, GFP reconstitution across synaptic partners; MB, mushroom body; nAChR, nicotinic acetylcholine receptor; RNAi, RNA interference 


\section{Abstract}

Short-term exposure to nicotine induces positive effects in mice, monkeys and humans, including mild euphoria, hyperactivity, and enhanced cognition. However, the underlying neural basis and molecular mechanisms for these effects remain poorly understood. Here, using a video recording system, we find that acute nicotine administration induces locomotor hyperactivity in Drosophila, similar to observations made in higher model organisms. Suppressing dopaminergic neurons or down-regulating dopamine 1-like receptor (DopR) abolishes this acute nicotine response, but surprisingly, does so only in male flies. Using a GFP reconstitution across synaptic partners (GRASP) approach, we show that dopaminergic neurons possess potential synaptic connections with acetylcholinergic neurons in wide regions of the brain. Furthermore, dopaminergic neurons are widely activated upon nicotine perfusion in both sexes, while the response curve differs significantly between the sexes. Moreover, knockdown of the $\beta 1$ nicotine acetylcholine receptor (nAChR) in dopaminergic neurons abolishes the acute nicotine response only in male flies, while panneural knock-down occurs in both sexes. Taken together, our results reveal that in fruit flies, dopaminergic neurons mediate nicotine-induced acute locomotor hyperactivity in a sexually dimorphic manner, and Drosophila $\beta 1 \mathrm{nAChR}$ subunit plays a crucial role in this nicotine response. These findings provide important insights into the molecular and neural basis of acute nicotine effects, and the underlying mechanisms may play conserved roles across species.

Keywords: nicotine, locomotor hyperactivity, Drosophila, dopaminergic neurons, 
sexual dimorphism, $\beta 1 \mathrm{nAChR}$

\section{INTRODUCTION}

Nicotine, a major compound found in tobacco, is the primary factor leading to addiction to smoking (Robinson and Pritchard, 1992, Benowitz, 2010). In mammals, repeated or long-term nicotine exposure results in a series of complicated changes at both molecular and behavioral levels, and may ultimately cause nicotine dependence (Markou, 2008, Ray et al., 2009, Subramaniyan and Dani, 2015). Among various nicotine effects, changes in locomotor activity have been frequently observed, thus it has been used as a main parameter to evaluate nicotine effects (Philpot et al., 2012). For example, both acute and chronic nicotine exposure induces locomotor hyperactivity in rats (Dwoskin et al., 1999).

Locomotor activity has recently been found to change upon nicotine exposure of fruit flies. In the startle-induced negative geotaxis assay using vertical columns, flies showed reduced climbing activity in response to nicotine treatment, and the acute and long-lasting effects were found to be mediated by dopaminergic system and cAMP/CREB pathway, respectively (Bainton et al., 2000, Hou et al., 2004). Using a video recording system where flies are tested in horizontal tubes (Zimmerman et al., 2008), our previous study revealed that chronic nicotine treatment induces locomotor hyperactivity in flies, and a protein in the decapping complex, decapping protein 2 , was found to be a key factor in mediating nicotine-induced locomotor hyperactivity 
(Ren et al., 2012). Early onset of hyperactivity upon nicotine exposure has been observed in flies, though the mechanistic details behind this observation have so far not been studied (Bainton et al., 2000). To access the acute hyperactivity of the acute nicotine response in flies, we adopted the same video recording system to analyze locomotion activity accurately.

Nicotinic acetylcholine receptors (nAChRs), are transmembrane oligomers consisting of five subunits (Cooper et al., 1991). In mammals, neuronal nAChRs are constituted by $\alpha$ - and $\beta$ - subunits ( $\alpha 2-\alpha 10$ and $\beta 2-\beta 4)$ (Millar, 2003) in various combinations. Widely distributed in the peripheral and central nervous system (Wu and Lukas, 2011), nAChRs play fundamental roles in the regulation of neural activity (Dani and Bertrand, 2007, Albuquerque et al., 2009). In particular, different types of nAChR subunits have been found to regulate certain effects of nicotine (Dupuis et al., 2012), e.g., the $\alpha 5 \mathrm{nAChR}$ subunit is involved in nicotine short-term effects in mice, and the $\beta 2 \mathrm{nAChR}$ subunit participates in the modulation of protective responses to nicotine stress in mice (Cohen et al., 2002, Salas et al., 2003). In Drosophila, ten nAChR subunits have been identified and designated as either $\alpha$-type $(\alpha 1-\alpha 7)$ or $\beta$-type $(\beta 1-\beta 3)$ (Sattelle et al., 2005), both of which are exclusively located in the central nervous system (Schuster et al., 1993). These receptors allow fast excitatory synaptic transmission (Campusano et al., 2007), and were shown to regulate sensory and cognitive processes (Dupuis et al., 2012).

In mammals, dopamine represents a reward signal and is crucial for the reinforcement that promotes the self-administration of nicotine (Nestler, 2005). Similarly, the 
nicotine response was also blocked in flies treated with dopamine inhibitor, suggesting a conserved role of dopaminergic system in mediating nicotine effects (Bainton et al., 2000). Here, we characterized the acute nicotine response of locomoter hyperactivity in flies. In addition, we found that dopaminergic neurons and the $\beta 1 \mathrm{nAChR}$ subunit play crucial roles in mediating this nicotine response.

\section{EXPERIMENTAL PROCEDURES}

\section{Fly strains}

Fruit fly strains were courtesy of the following groups/researchers: Dumb- by Dr. F. W. Wolf (Ernest Gallo Clinic and Research Center); c5-Gal4, c205-Gal4, c232-Gal4, and c819-Gal4 by Dr. L. Liu (Institute of Biophysics); UAS-GCaMP3 by Dr. L. L. Looger (Howard Hughes Medical Institute, Janelia Farm Research Campus); 247-Gal4 by Dr.

M. Heisenberg (University of Würzburg); Cha-LexA by Dr. M. Landgraf (Cambridge United Kingdom); TH-Gal4 by Dr. J. Hirsh (University of Virginia); Trh-Gal4 and UAS-shibire $^{t s}$ by Dr. Y. Rao (Peking University), LexAop-CD4::GPF11; UAS-CD4::GFP1-10 by K. Scott (University of California); and PL00420/TM3Sb, elav-Gal4 and OK107-Gal4 by the Bloomington Drosophila Stock Center. UAS-DopR-RNAi (RNA interference) (107058) was provided by the Vienna Drosophila RNAi Center; RNAi fly strains for nAChR $\alpha 1$, nAChR 22 , nAChR $\alpha 3$, nAChR $\alpha 4, \mathrm{nAChR} \alpha 5, \mathrm{nAChR} \alpha 6, \mathrm{nAChR} \alpha 7, \mathrm{nAChR} \beta 1, \mathrm{nAChR} \beta 2$, and nAChR $\beta 3$ were obtained from the Tsinghua fly center. Flies were raised on Bloomington Drosophila Stock Center standard medium at $25{ }^{\circ} \mathrm{C}$ and $60 \%$ relative humidity under $12 \mathrm{~h} / 12 \mathrm{~h}$ light/dark (LD) conditions. Flies were collected at eclosion and aged for 3-4 
days before experiments.

\section{Behavior assay}

Individual flies were placed into $65 \mathrm{~mm} \times 5 \mathrm{~mm}$ monitor tubes with $2 \%$ agar food at ZT 12 , and $2 \mu 1$ liquid food containing $5 \%$ yeast and $5 \%$ sucrose as the normal food was delivered to the tube at ZT 2. For experimental groups, nicotine (Sigma, N3876) was added to the normal food at given concentrations. Locomotor activity was monitored in the LD cycle using the video recording system (Zimmerman et al., 2008). Web cameras with a $640 \times 480$ resolution were pretreated to allow reception of infrared light. Images were acquired every $1 \mathrm{sec}$ and further processed by Pysolo24 (Gilestro and Cirelli, 2009) to determine the location of the flies. The total walking distance was calculated to represent locomotor activity.

\section{Food-intake assay}

To examine the food intake in our setup, flies were entrained in the recording tubes with the same paradigm above. Brilliant Blue (Care, Chemodist Industris) was added to the food to a final concentration of $1 \%$ before fed to flies for $15 \mathrm{~min}$. According to a standard method (Edgecomb et al., 1994), flies were frozen on dry ice immediately and homogenized in PBS buffer and centrifuged (12000 rpm) for 30min. Supernatants were transferred to a new tube, and its absorbance was measured in the cuvettes at $625 \mathrm{~nm}$ to reflect the amount of food consumption.

\section{GRASP experiments}

GRASP experiments were performed according to a standard method (Feinberg et al., 2008). Briefly, the two parts of the split-GFP were separately expressed in two types 
of neurons, using the UAS/Gal4 and the LexA/LexAop dual expression systems. We constructed the flies of LexAop-CD4::GPF11/+;TH-Gal4,UAS-CD4::GFP1-10/ Cha-LexA, in which CD4::spGFP11 was expressed under the control of Cha-LexA (labeling cholinergic neurons), and CD4::spGFP1-10 was expressed under the control of TH-Gal4 (labeling dopaminergic neurons), respectively.

\section{Immunohistochemistry and Imaging}

Brains of adult fruit flies were processed according to a standard immunohistochemistry protocol (Wolff et al., 2014). Briefly, we dissected the brains in cold phosphate buffered saline (PBS, containing $1.86 \mathrm{mM} \mathrm{NaH} \mathrm{PO}_{4}, 8.41 \mathrm{mM}$ $\mathrm{NaH}_{2} \mathrm{PO}_{4}$, and $175 \mathrm{mM} \mathrm{NaCl}$ ) and fixed them in 4\% paraformaldehyde in PBST $(0.3 \%$ Triton in PBS) at room temperature for $40 \mathrm{~min}$. After three 20 -min washes in PBST brain samples were blocked in $5 \%$ NGS for $1 \mathrm{~h}$ at $25^{\circ} \mathrm{C}$, then incubated in mouse antibody against FasII (1:200, DSHB) (Fushima and Tsujimura, 2007) overnight at $4^{\circ} \mathrm{C}$. After three 20 -min washes, a goat anti-mouse Alexa 555 secondary antibody (1:500, Invitrogen) was applied overnight at $4^{\circ} \mathrm{C}$. Brain samples were imaged using a Leica SP5 II confocal microscope and Z stack images were scanned at $1-\mu \mathrm{m}$ section intervals with a resolution of $1,024 \mathrm{X} 1,024$ pixels. Confocal stacks were analyzed with Image J (National Institutes of Health).

\section{Calcium Imaging with Nicotine Perfusion}

Flies of TH-Gal4; GCaMP3.0 were anesthetized on ice, and fly brains were dissected gently in saline (Wilson et al., 2004). Brain samples were pre-incubated in $1 \mu \mathrm{M}$ TTX 
(KangTe, 120516) in saline for 10 minutes, followed by 1 min nicotine $(0.6 \mathrm{mM})$ perfusion. Images were obtained using a Leica SP5 II confocal microscope with a 20 $\mathrm{X}$ water objective (numerical aperture 1.00). The resolution of the images was $256 \times$ 256 pixels; photos were taken at a rate of 1 frame per second for 300 seconds. Region of interest selection and quantification of fluorescent intensity was performed using Leica LAS-AF software package. The neural activity of dopaminergic neurons were analyzed in the brain regions of the mushroom body (MB) vertical lobe, ellipsoid bod y (EB) and fan-shaped body $(\mathrm{FB})$. We calculated the baseline fluorescence $\left(\mathrm{F}_{0}\right)$ by averaging the calcium signal in 10 frames prior to nicotine delivery. For each brain sample, to calculate the $\Delta \mathrm{F} / \mathrm{F}$, the change of fluorescence $(\Delta \mathrm{F})$ for each time point was divided by $\mathrm{F}_{0}$. The peak response $(\max \Delta \mathrm{F} / \mathrm{F})$ of each brain was calculated as the average of $\Delta \mathrm{F} / \mathrm{F}$ during the 10 frames on both sides of the maximum response.

\section{Real-Time Quantitative PCR}

Total RNA was extracted from approximately 100 fly heads using Trizol reagent (Invitrogen). All RNA samples were treated with RNase-free DNase (Promega), and cDNA was synthesized using a high capacity cDNA reverse transcription kit (Takara). The mRNA levels of different genes were measured using SYBR-GREEN PCR Mix (Takara) on an ABI 7500 Sequence Detection System (Applied Biosystems). The relative mRNA levels were calculated using the comparative delta $\mathrm{C}_{\mathrm{T}}$ method. $\mathrm{Rp} 49$ was used as a reference gene. Primers for rp49 were designed with the following sequences: 5, primer, AAGCGTCAACTGTTCCATAGCC-; 3' primer, TGCGCCTTAGCTGCCTTAAGT; and for DopR: 5' primer, 
ATCATGGGCGTTTTTCTCATC ; 3’ primer, AGGTGAGGATCTTGAAGGTCTGT .

\section{Statistical analysis}

SPSS (SPSS, Inc.) was used for data analysis. For behavioral experiments and calcium imaging experiments, independent samples t-test was performed for comparison between two groups. Data are presented as mean behavioral responses, with error bars representing the standard error of the mean (SEM). Differences between groups were considered significant if the probability of error was less than $0.05(\mathrm{P}<0.05)$

\section{RESULTS}

\section{Nicotine administration induces acute locomotor hyperactivity in Drosophila}

In a previous study, nicotine exposure was reported to impair the ability of negative geotaxis of Drosophila in a dose-dependent manner, while the authors also observed a rapid response of hyperactivity (Bainton et al., 2000). To analyze this acute effect of nicotine, we adopted a video monitoring system (Zimmerman et al., 2008) to accurately record the locomotor activity. In our behavioral assay, flies were placed individually in recording tubes (Fig. 1a), and the starting time point of recording was ZT 0 . In the first 2 hours from ZT 0 to ZT 2, we observed that the locomotor activity gradually decreased (Fig. 1b-e), a result in agreement with the activity rhythm of flies (Chiu et al., 2010). Liquid food was supplied at ZT 2, and the locomotor activity was dramatically reduced in normal food groups. However, when $0.6 \mathrm{mM}$ nicotine was added, the locomotor activity remained at a higher level for more than 1 hour in both 
males and females of two wild type strains ( $w^{1118}$ and CS) we examined (Fig. 1b-e). We then quantified the difference of the locomotor activity within 1 hour after the treatment between the nicotine group and the control group. As shown in Figure 2, flies in $0.6 \mathrm{mM}$ nicotine groups exhibited a significant increase in locomotor activity when compared to normal food control groups (Fig. 2a-d).

To test the dosage dependent effects of nicotine on locomotor activity, we tested with four concentrations in addition to $0.6 \mathrm{mM}$ of nicotine. In our previous study, we reported that $3.0 \mathrm{mM}$ nicotine in food induced chronic locomotor hyperactivity (Ren et al., 2012). In this study, we found that acute treatment of nicotine at $3.0 \mathrm{mM}$ induced locomotor hyperactivity in female flies, not however, in male flies. In addition, nicotine-containing food at concentrations lower than $3.0 \mathrm{mM}$ consistently induced locomotor hyperactivity in both male and female flies (Fig. 2a-d). In contrast, upon treatment with nicotine at concentrations higher than $3.0 \mathrm{mM}$, these flies lacked locomotor hyperactivity, except that $w^{1118}$ female flies showed an increase in locomotor activity when treated with $4.2 \mathrm{mM}$ nicotine (Fig. 2d). Moreover, locomotor activity was decreased in $w^{1118}$ male flies (Fig. 2c), suggesting that high dose nicotine treatment is toxic for flies. Therefore, we used $0.6 \mathrm{mM}$ nicotine in all subsequent experiments. These results indicate that nicotine exposure at low concentration $(0.6$ $\mathrm{mM}$ ) induces locomotor hyperactivity in both male and female flies, while their response patterns are different between sexes.

We further measured the food intake of flies with the same treatment in the locomotion assay. The result showed that compared to that in the normal food group, 
the food intake was reduced in the $0.6 \mathrm{mM}$ nicotine group, and it was even less in the $6.0 \mathrm{mM}$ nicotine group (Fig. 2e-f). Notably, locomotor hyperactivity was observed in the $0.6 \mathrm{mM}$ group but not in the $6 \mathrm{mM}$ group, which suggested that a reduction in food intake was not the main cause of the increase in locomotor activity.

\section{Dopaminergic system is required for acute locomotor hyperactivity induced by}

\section{nicotine administration in male adult flies}

Activation of nicotinic cholinergic receptors facilitates the release of a variety of neurotransmitters in mammals (Dajas-Bailador and Wonnacott, 2004). To investigate which neurotransmitters are required for nicotine-induced acute hyperactivity in flies, we individually blocked dopaminergic, octopaminergic and serotonergic neurons by

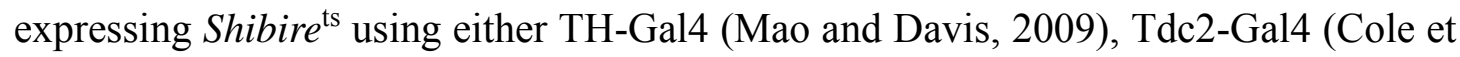
al., 2005) or Trh-Gal4 (Alekseyenko et al., 2010). Shibire ${ }^{\text {ts }}$ is a temperature-sensitive dynamin protein, with which the synaptic output is blocked at restrictive temperatures (Kitamoto, 2001). We found that blocking dopaminergic neurons inhibited acute nicotine-induced locomotor hyperactivity in male flies, not however, in female flies. In contrast, repressing octopaminergic and serotonergic neurons did not affect the acute nicotine response in either male or female groups (Fig. 3a-b). At a permissive temperature $\left(25^{\circ} \mathrm{C}\right)$, when the neurotransmission is not affected by Shibire ${ }^{\text {ts }}$, male and female flies still displayed locomotor hyperactivity upon nicotine treatment (Fig. $3 c-d)$. In summary, our results provide strong evidence that dopaminergic neurons play an essential role in mediating nicotine-induced acute hyperactivity in male flies. 
DopR is a type 1 dopaminergic receptor belonging to the G-protein coupled receptor family, and has been reported to play critical roles in regulating locomotor activity and mediating drug effect in Drosophila (Rothenfluh and Heberlein, 2002). To investigate whether $D o p R$ is essential for acute locomotor hyperactivity induced by nicotine administration, we examined the nicotine response of two DopR mutants, PL00420 and Dumb: We found that the acute nicotine effect was abolished in male but not female flies of these two mutant DopR lines (Fig. 4a-b), which is consistent with the effect of blocking dopaminergic neurons. In addition, we knocked down DopR expression with DopR-RNAi in the nervous system using elav-Gal4. In these flies, the acute nicotine effect was also diminished in male flies (Fig. 4c). In contrast, when DopR was knocked down in individual brain regions using c205-Gal4 and c5-Gal4 for the FB (Liu et al., 2006), OK107-Gal4 (Kobayashi et al., 2006) and MB247-Gal4 (Zhang et al., 2007) for the MB, and c819-Gal4 and c232-Gal4 (Kong et al., 2010) for the EB, nicotine was still able to induce hyperactivity (Fig. 4c). Using a qPCR approach, we demonstrated that DopR expression in the two mutants and DopR-RNAi knockdown flies driven by elav-Gal4 was significantly reduced (Fig. 4d-f). Taken together, these results indicate that dopaminergic system plays important roles in acute nicotine response in male flies.

\section{Direct activation of dopaminergic neurons by nicotine perfusion}

In flies, nicotine activates neurons through nAChRs, which are receptors for endogenous neural transmitter acetylcholine. In mammals, nAChRs are abundantly 
expressed in dopaminergic neurons and located at both somatic and terminal sites, where they are critically involved in nicotine addiction (Nisell et al., 1994, Champtiaux et al., 2003). To investigate whether dopaminergic neurons also display a wide distribution of $\mathrm{nAChR}$ in Drosophila, we first examined the potential connections between acetylcholinergic neurons and dopaminergic neurons with the GRASP approach (Feinberg et al., 2008). In flies with two parts of GFP expressed separated in these two types of neurons (see methods for details), strong GRASP signals were detected in several brain regions, including in the $\mathrm{MB}, \mathrm{EB}$ and $\mathrm{FB}$ in both male and female flies (Fig. 5a-f). In addition, we constructed a negative control, where flies contained all of the transgenes except Cha-LexA. There, we no GRASP signal was observed (data not show). Together, our results suggest that there are wide connections between dopaminergic neurons and the acetylcholinergic neurons, implying that nAChRs are expressed and located on the post-synaptic sites of dopaminergic neurons, thus these neurons are capable to respond to nicotine directly.

To confirm this speculation, we performed nicotine perfusion and functional calcium imaging in dopaminergic neurons. To record the direct neural response to nicotine, we incubated all brain samples with $1 \mu \mathrm{M}$ neurotoxin tetrodotoxin (TTX) for 10 minutes to block action potential. In the three brain regions exhibiting strong GRASP signal, the $\mathrm{MB}$, the $\mathrm{EB}$ and the $\mathrm{FB}$, the calcium signal was significantly increased upon nicotine perfusion in both male and female flies (Fig. 6). Moreover, we found that the nicotine response in female flies was stronger than that in male flies (Fig. 6g). We further analyzed the response curve by calculating the time duration for the signal to 
reach the maximal value from the half value $\left(\mathrm{t}_{0}\right)$ and the time duration for the signal to reduce from the maximal value to the half value $\left(t_{1}\right)$. The results showed that $t_{0}$ is longer in female than male flies in $\mathrm{EB}$ and $\mathrm{FB}$ regions, but not in the $\mathrm{MB}$, while $\mathrm{t}_{1}$ is longer in female than male flies in all three brain regions (Fig. 6h-i). Taken together, dopaminergic neurons can be directly and significantly activated by nicotine exposure, and the nicotine response in these neurons are different between sexes.

\section{The $\beta 1 \mathrm{nAChR}$ subunit in dopaminergic neurons is required for nicotine-induced}

\section{acute locomotor hyperactivity in male flies}

nAChRs are widely expressed in the central nervous system, where they supply fast synaptic excitatory transmission and have crucial roles in behavioral and cognitive functions (Dupuis et al., 2012). In Drosophila, it has been reported that the $\alpha 7 \mathrm{nAChR}$ subunit is required for escape behavior and chronic nicotine-induced locomotor hyperactivity (Fayyazuddin et al., 2006, Ren et al., 2012). To determine which subunit mediates the acute nicotine effect, we knocked down the expression of ten nAChR subunits by individually expressing the RNAi constructs in dopaminergic neurons under the control of TH-Gal4. In all ten fly lines examined, the acute nicotine-induced locomotor hyperactivity was only repressed in the male flies with $\beta 1 \mathrm{nAChR}$ knocked-down (Fig. 7a-b). Furthermore, pan-neuronal knockdown of $\beta 1 \mathrm{nAChR}$ by elav-Gal4 resulted in complete lack of hyperactivity, both in male and female flies (Fig. 7c). Together, these findings reveal that the $\beta 1 \mathrm{nAChR}$ subunit is required for the nicotine-induced locomotor hyperactivity in both sexes, while in male flies, its 
function in dopaminergic neurons plays a critical role.

\section{DISCUSSION}

In this study, we found that nicotine administration induces acute locomotor hyperactivity in wild type flies, with sexual differences related to the involvement of dopaminergic system. Furthermore, the $\beta 1 \mathrm{nAChR}$ subunit plays a critical role in mediating this acute nicotine effect in both genders, while its function in male flies was also mainly carried out in dopaminergic neurons. Sexual dimorphism in nicotine responses has been widely observed across species. In humans, upon an acute administration of nicotine, shorter reaction time and more accurate measurement in the visual-spatial tasks were detected in male when compared to female (Neumann et al., 2007). In addition, rhesus monkeys also showed sexual differences in a memory-related task after low-dose nicotine-intake (Buccafusco et al., 1999). Similarly, active avoidance learning in rats was found to have improved upon nicotine treatment in male, while impaired in females (Yilmaz et al., 1997). In agreement with these results, neural activity in the brain was differentially affected following acute nicotine treatment in male and female mice (Amann et al., 2008). Interestingly, we also observed sexual dimorphism in nicotine-induced behavioral changes in Drosophila. Upon nicotine administration, female flies of both wild type strains exhibited hyperactivity in 3 dosage groups $(0.6,1.8$, and $3.0 \mathrm{mM})$, whereas male flies failed to display hyperactivity in the $3.0 \mathrm{mM}$ group. Moreover, blocking the output of dopaminergic neurons or knock-down of DopR abolished the acute nicotine effect in 
male flies, not, however, in females. Furthermore, in the functional calcium imaging experiments, the nicotine response of dopaminergic neurons was also sexually dimorphic. Therefore, we hypothesize that the nicotine-induced acute hyperactivity is primarily mediated by dopaminergic neurons in male flies, while more than one type of neurons coordinately regulate this effect in female flies. It will be of considerable importance to determine in future studies whether such difference also exists in mammals.

Different types of nAChR subunits have been reported to be expressed in several brain regions, where they fulfil different functions in sensory and cognitive processes in the central nervous system (Dupuis et al., 2012). The Drosophila genome contains ten $\mathrm{nAChR}$ genes, of which one $\mathrm{D} \alpha 7$ subunit is found to be involved in regulating behavior (Fayyazuddin et al., 2006, Ren et al., 2012). Here, we revealed that $\beta 1$ $\mathrm{nAChR}$ in dopaminergic neurons is the key subunit for acute locomotor hyperactivity induced by nicotine administration. No such role, however, has been identified for the $\alpha 7$ nAChR subunit, suggesting that different phases of nicotine effects may be attributed to different molecular and neural pathways. In mammals, it was reported that nicotine diminished episodic hypoxia-induced protective breathing and arousal responses though $\beta 2 \mathrm{nAChR}$ subunit in mice (Cohen et al., 2002). In light of our findings, we propose that $\beta$-nAChR subunits play essential roles in acute nicotine responses.

In a previous study on acute ethanol response, DopR was reported to mediate locomotor hyperactivity induced by ethanol exposure. Furthermore, a subset of 
dopaminergic neurons projecting to the central complex is responsible for this ethanol effect (Kong et al., 2010). In our study, we found that DopR was also required for acute nicotine response in male flies. However, pan-neuronal knockdown of DopR expression eliminated the acute hyperactivity effect, whereas down-regulating DopR in individual brain regions, like the $\mathrm{MB}$, the $\mathrm{EB}$ and the $\mathrm{FB}$, did not abolish this increase of locomotor activity. In agreement with these observations, our calcium imaging data show that nicotine exposure triggers wide activation of dopaminergic neurons in different brain regions. These results indicate that in contrast to ethanol consumption, nicotine exposure induces wide activation of dopaminergic neurons, which coordinately contribute to the behavioral changes of hyperactivity. 


\section{References}

Albuquerque EX, Pereira EFR, Alkondon M, Rogers SW (2009) Mammalian Nicotinic Acetylcholine Receptors: From Structure to Function. Physiol Rev 89:73-120.

Alekseyenko OV, Lee C, Kravitz EA (2010) Targeted Manipulation of Serotonergic Neurotransmission Affects the Escalation of Aggression in Adult Male Drosophila melanogaster. PloS one 5.

Amann LC, Phillips JM, Halene TB, Siegel SJ (2008) Male and Female Mice Differ for Baseline and Nicotine-Induced Event Related Potentials. Behavioral neuroscience 122:982-990.

Bainton RJ, Tsai LTY, Singh CM, Moore MS, Neckameyer WS, Heberlein U (2000) Dopamine modulates acute responses to cocaine, nicotine and ethanol in Drosophila. Current Biology 10:187-194.

Benowitz NL (2010) Nicotine addiction. The New England journal of medicine 362:2295-2303.

Buccafusco JJ, Jackson WJ, Jonnala RR, Terry AV (1999) Differential improvement in memory-related task performance with nicotine by aged male and female rhesus monkeys. Behav Pharmacol 10:681-690.

Campusano JM, Su H, Jiang SA, Sicaeros B, O'Dowd DK (2007) nAChR-Mediated calcium responses and plasticity in Drosophila Kenyon cells. Dev Neurobiol 67:1520-1532.

Champtiaux N, Gotti C, Cordero-Erausquin M, David DJ, Przybylski C, Lena C, Clementi F, Moretti M, Rossi FM, Le Novere N, McIntosh JM, Gardier AM, Changeux JP (2003) Subunit composition of functional nicotinic receptors in dopaminergic neurons investigated with knock-out mice. Journal of Neuroscience 23:7820-7829.

Chiu JC, Low KH, Pike DH, Yildirim E, Edery I (2010) Assaying locomotor activity to study circadian rhythms and sleep parameters in Drosophila. Journal of visualized experiments : JoVE.

Cohen G, Han ZY, Grailhe R, Gallego J, Gaultier C, Changeux JP, Lagercrantz H (2002) beta 2 nicotinic acetylcholine receptor subunit modulates protective responses to stress: A receptor basis for sleep-disordered breathing after nicotine exposure. Proceedings of the National Academy of Sciences of the United States of America 99:13272-13277.

Cole SH, Carney GE, McClung CA, Willard SS, Taylor BJ, Hirsh J (2005) Two functional but noncomplementing Drosophila tyrosine decarboxylase genes. Journal of Biological Chemistry 280:14948-14955.

Cooper E, Couturier S, Ballivet M (1991) Pentameric Structure and Subunit Stoichiometry of a Neuronal Nicotinic Acetylcholine-Receptor. Nature 350:235-238.

Dajas-Bailador F, Wonnacott S (2004) Nicotinic acetylcholine receptors and the regulation of neuronal signalling. Trends in pharmacological sciences 25:317-324.

Dani JA, Bertrand D (2007) Nicotinic acetylcholine receptors and nicotinic cholinergic mechanisms of the central nervous system. Annu Rev Pharmacol 47:699-729.

Dupuis J, Louis T, Gauthier M, Raymond V (2012) Insights from honeybee (Apis mellifera) and fly (Drosophila melanogaster) nicotinic acetylcholine receptors: from genes to behavioral functions. Neuroscience and biobehavioral reviews 36:1553-1564.

Dwoskin LP, Crooks PA, Teng LH, Green TA, Bardo MT (1999) Acute and chronic effects of nornicotine on locomotor activity in rats: altered response to nicotine. Psychopharmacology 145:442-451.

Edgecomb RS, Harth CE, Schneiderman AM (1994) Regulation of Feeding-Behavior in Adult Drosophila-Melanogaster Varies with Feeding Regime and Nutritional State. Journal of Experimental Biology 197:215-235.

Fayyazuddin A, Zaheer MA, Hiesinger PR, Bellen HJ (2006) The Nicotinic Acetylcholine Receptor Da7 Is 
Required for an Escape Behavior inDrosophila. Plos Biol 4:e63.

Feinberg EH, VanHoven MK, Bendesky A, Wang G, Fetter RD, Shen K, Bargmannl Cl (2008) GFP reconstitution across synaptic partners (GRASP) defines cell contacts and Synapses in living nervous systems. Neuron 57:353-363.

Fushima K, Tsujimura H (2007) Precise control of fasciclin II expression is required for adult mushroom body development in Drosophila. Dev Growth Differ 49:215-227.

Gilestro GF, Cirelli C (2009) pySolo: a complete suite for sleep analysis in Drosophila. Bioinformatics 25:1466-1467.

Hou J, Kuromi H, Fukasawa Y, Ueno K, Sakai T, Kidokoro Y (2004) Repetitive exposures to nicotine induce a hyper-responsiveness via the cAMP/PKA/CREB signal pathway in Drosophila. Journal of neurobiology 60:249-261.

Kitamoto T (2001) Conditional modification of behavior in drosophila by targeted expression of a temperature-sensitive shibire allele in defined neurons. Journal of neurobiology 47:81-92.

Kobayashi M, Michaut L, Ino A, Honjo K, Nakajima T, Maruyama Y, Mochizuki H, Ando M, Ghangrekar I, Takahashi K, Saigo K, Ueda R, Gehring WJ, Furukubo-Tokunaga K (2006) Differential microarray analysis of Drosophila mushroom body transcripts using chemical ablation. Proceedings of the National Academy of Sciences of the United States of America 103:14417-14422.

Kong EC, Woo K, Li HY, Lebestky T, Mayer N, Sniffen MR, Heberlein U, Bainton RJ, Hirsh J, Wolf FW (2010) A Pair of Dopamine Neurons Target the D1-Like Dopamine Receptor DopR in the Central Complex to Promote Ethanol-Stimulated Locomotion in Drosophila. PloS one 5.

Liu G, Seiler H, Wen A, Zars T, Ito K, Wolf R, Heisenberg M, Liu L (2006) Distinct memory traces for two visual features in the Drosophila brain. Nature 439:551-556.

Mao Z, Davis RL (2009) Eight different types of dopaminergic neurons innervate the Drosophila mushroom body neuropil: anatomical and physiological heterogeneity. Frontiers in neural circuits $3: 5$.

Markou A (2008) Neurobiology of nicotine dependence. Philos T R Soc B 363:3159-3168.

Millar NS (2003) Assembly and subunit diversity of nicotinic acetylcholine receptors. Biochem Soc T 31:869-874.

Nestler EJ (2005) Is there a common molecular pathway for addiction? Nature neuroscience 8:1445-1449.

Neumann DL, Fitzgerald ZT, Furedy JJ, Boyle GJ (2007) Sexually dimorphic effects of acute nicotine administration on arousal and visual-spatial ability in non-smoking human volunteers. Pharmacol Biochem Be 86:758-765.

Nisell M, Nomikos GG, Svensson TH (1994) Systemic Nicotine-Induced Dopamine Release in the Rat Nucleus-Accumbens Is Regulated by Nicotinic Receptors in the Ventral Tegmental Area. Synapse 16:36-44.

Philpot RM, Engberg ME, Wecker L (2012) Effects of nicotine exposure on locomotor activity and pCREB levels in the ventral striatum of adolescent rats. Behavioural brain research 230:62-68.

Ray R, Schnoll RA, Lerman C (2009) Nicotine Dependence: Biology, Behavior, and Treatment. Annu Rev Med 60:247-260.

Ren J, Sun J, Zhang Y, Liu T, Ren Q, Li Y, Guo A (2012) Down-regulation of Decapping Protein 2 mediates chronic nicotine exposure-induced locomotor hyperactivity in Drosophila. PloS one 7:e52521.

Robinson JH, Pritchard WS (1992) The Role of Nicotine in Tobacco Use. Psychopharmacology 
108:397-407.

Rothenfluh A, Heberlein U (2002) Drugs, flies, and videotape: the effects of ethanol and cocaine on Drosophila locomotion. Current opinion in neurobiology 12:639-645.

Salas R, Orr-Urtreger A, Broide RS, Beaudet A, Paylor R, De Biasi M (2003) The nicotinic acetylcholine receptor subunit $\alpha 5$ mediates short-term effects of nicotine in vivo. Molecular pharmacology 63:1059-1066.

Sattelle DB, Jones AK, Sattelle BM, Matsuda K, Reenan R, Biggin PC (2005) Edit, cut and paste in the nicotinic acetylcholine receptor gene family of Drosophila melanogaster. Bioessays 27:366-376.

Schuster R, Phannavong B, Schroder C, Gundelfinger ED (1993) Immunohistochemical Localization of a Ligand-Binding and a Structural Subunit of Nicotinic Acetylcholine-Receptors in the Central-Nervous-System of Drosophila-Melanogaster. Journal of Comparative Neurology 335:149-162.

Subramaniyan M, Dani JA (2015) Dopaminergic and cholinergic learning mechanisms in nicotine addiction. Addiction Reviews 2015 1349:46-63.

Wilson RI, Turner GC, Laurent G (2004) Transformation of olfactory representations in the Drosophila antennal lobe. Science 303:366-370.

Wolff SBE, Grundemann J, Tovote P, Krabbe S, Jacobson GA, Muller C, Herry C, Ehrlich I, Friedrich RW, Letzkus JJ, Luthi A (2014) Amygdala interneuron subtypes control fear learning through disinhibition. Nature 509:453-+.

Wu J, Lukas RJ (2011) Naturally-expressed nicotinic acetylcholine receptor subtypes. Biochemical pharmacology 82:800-807.

Yilmaz O, Kanit L, Okur BE, Pogun S (1997) Effects of nicotine on active avoidance learning in rats: Sex differences. Behav Pharmacol 8:253-260.

Zhang K, Guo JZ, Peng Y, Xi W, Guo A (2007) Dopamine-mushroom body circuit regulates saliency-based decision-making in Drosophila. Science 316:1901-1904.

Zimmerman JE, Raizen DM, Maycock MH, Maislin G, Pack Al (2008) A Video Method to Study Drosophila Sleep. Sleep 31:1587-1598. 


\section{Acknowledgements}

We thank Dr. Y. Rao and Dr. M. Landgraf, as well as the Bloomington Stock center and Tsinghua fly center for fly stocks. We are grateful to Z. Zhang, X. Li and N. Chen for manuscript discussion. We thank H. Lu, J. Hou, and M. Zhou for technical support during the preparation of experiment. This work was supported by grants from the National Science Foundation of China (91132709, 31130027, and 31571089), the 973 Program (2011CBA00400), One Hundred Talent Project of Chinese Academy of Science (KSCX2-YW-R-156), and the "Strategic Priority Research Program" (XDB02040100).

\section{Figure legends}

Fig. 1 Nicotine induced acute locomotor hyperactivity in Drosophila

(a) Schematic diagram of behavioral assay. (b-e) The time course of locomotor activity in response to $0.6 \mathrm{mM}$ nicotine administration. In both WT flies strains (CS and $w^{1118}$ ), flies with nicotine food display higher locomotor activity than those supplied with normal food. In the line graph, each point represents walking distance in $5 \mathrm{~min}$. The gray bars indicate the time window of 1 hour for calculating the cumulative activity.

Fig. 2 Locomotor activity of flies changes upon nicotine administration at different concentrations

(a-d) Locomotor activity of CS and $w^{1118}$ WT flies treated with 0.6, 1.8, 3.0, 4.2 and 
$6.0 \mathrm{mM}$ of nicotine in normal food. The $0 \mathrm{mM}$ group is the control group with normal food only. (e-f) Compared to normal food group, both male and female flies have less food intake in $0.6 \mathrm{mM}$ nicotine group, and even less food intake in $6 \mathrm{mM}$ nicotine group. Independent samples t-test was performed for comparison between two groups. $\mathrm{n}>100$ in each group. ${ }^{*}, \mathrm{p}<0.05 ; * *, \mathrm{p}<0.01 ; * * *, \mathrm{p}<0.001$. N.S. indicates no significant difference.

Fig. 3 Dopaminergic neurons are required for nicotine-induced locomotor hyperactivity in male flies

(a) At a restrictive temperature $\left(30^{\circ} \mathrm{C}\right)$, blocking dopaminergic neurons by expressing Shibire $^{\text {ts }}$ with TH-Gal4 inhibits nicotine induced hyperactivity in male flies, however, not when blocking octopaminergic and serotonergic neurons with Tdc2-Gal4 and Trh-Gal4, respectively. (b) Nicotine induced hyperactivity is not affected when blocking dopaminergic, octopaminergic, or serotonergic neurons in female flies. (c-d) At a permissive temperature $\left(25^{\circ} \mathrm{C}\right)$, both male and female flies show acute locomotor hyperactivity upon nicotine administration. Independent samples t-test was performed for comparison between two groups. $\mathrm{n}>50$ in each group. ${ }^{* * *}, \mathrm{p}<0.001$. N.S. indicates no significant difference.

Fig. 4 DopR is required for acute locomotor hyperactivity induced by nicotine administration in male flies

(a-b) In two DopR mutant strains, Dumb- (a) and PL00420 (b), locomotor activity of 
female flies significantly increases upon $0.6 \mathrm{mM}$ nicotine treatment, while it remains unchanged in male flies. (c-d) In both Dumb ${ }^{-}$and PL00420 mutant flies, the DopR expression level is significantly reduced. (e) The nicotine-induced acute locomotor hyperactivity is abolished when knocking-down DopR expression in the entire nervous system by elav-Gal4, but not when knocking-down it in separate brain regions. (f) In DopR knockdown flies driven by elav-Gal4, the DopR expression level was significantly reduced. Independent samples t-test was performed for comparison between two groups. $\mathrm{n}>50$ in each group. ${ }^{* *}, \mathrm{p}<0.01 ; * *, \mathrm{p}<0.001$. N.S. indicates no significant difference.

Fig. 5 GRASP signals between the acetylcholinergic neurons and dopaminergic neurons in the $\mathrm{MB}, \mathrm{EB}$ and $\mathrm{FB}$ brain regions

A representative brain of a male (upper) or a female (lower) fly with the genotype of LexAop-CD4::GPF11/+; TH-Gal4,UAS-CD4::GFP1-10/Cha-LexA. GRASP signal (green) is detected in several brains regions, including the $\mathrm{MB}, \mathrm{EB}$, and $\mathrm{FB}$. Particularly in the MB, GRASP signal is rich in the heel and the stalk of the vertical lobes. Neuropil is staining by FasII, shown in magenta. Scale bars represent $20 \mu \mathrm{m}$ in all panels.

Fig. 6 Dopaminergic neurons are activated by nicotine perfusion

(a-f) Nicotine perfusion induces a significant increase of calcium signal in dopaminergic neurons. The genotype is TH-Gal4;GCaMP3.0 in both male (a-c) and 
female (d-f) flies. The dotted lines in the insert picture show the recording regions at the MB vertical lobe ( $a$ and $d$ ), the EB ( $b$ and e), and the FB (c and f). Scale bars represent $20 \mu \mathrm{m}$. The time duration $t_{0}$ and $t_{1}$ are indicated in panel a. $(\mathrm{g})$ The maximum response to nicotine perfusion is significantly higher in female than male flies. Medians are shown as crosslines; $50 \%$ of the values are located within the box; whiskers represent the range of entire set of data. (h) In the EB and FB regions, $t_{0}$ is shorter in female flies than that in male flies, while it is comparable between sexes in the MB region. (i) In all three brain regions, $t_{1}$ is shorter in female flies than that in male flies. Independent samples t-test was performed for comparison between two groups. $\mathrm{n}=4-5$ in each group. ${ }^{*}, \mathrm{p}<0.05 ;{ }^{* *}, \mathrm{p}<0.01{ }^{* * *}, \mathrm{p}<0.001$. N.S. indicates no significant difference.

Fig. 7 The $\beta 1$ nAChR subunit plays a critical role in nicotine-induced acute hyperactivity

(a-b) Nicotine-induced locomotor hyperactivity is abolished when the $\beta 1 \mathrm{nAChR}$ subunit is knocked down in dopaminergic neurons in male (a) but not female (b) flies. Knock down of other nAChR subunits has no effect. (c) Panneural knock down of the $\beta 1 \mathrm{nAChR}$ results in the failure of acute nicotine response in both male and female flies. Independent samples t-test was performed for comparison between two groups. $\mathrm{n}>50$ in each group. $*, \mathrm{p}<0.05 ; * *, \mathrm{p}<0.01 ; * * *, \mathrm{p}<0.001$. N.S. indicates no significant difference. 

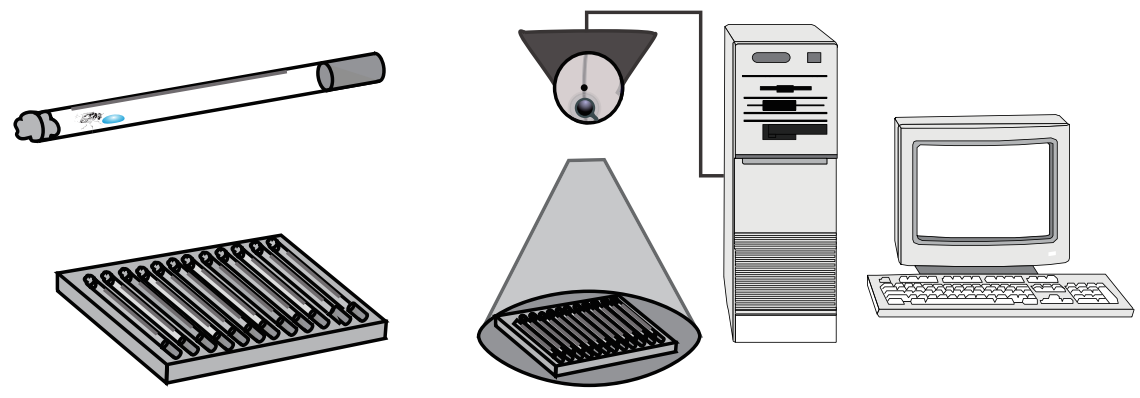

B
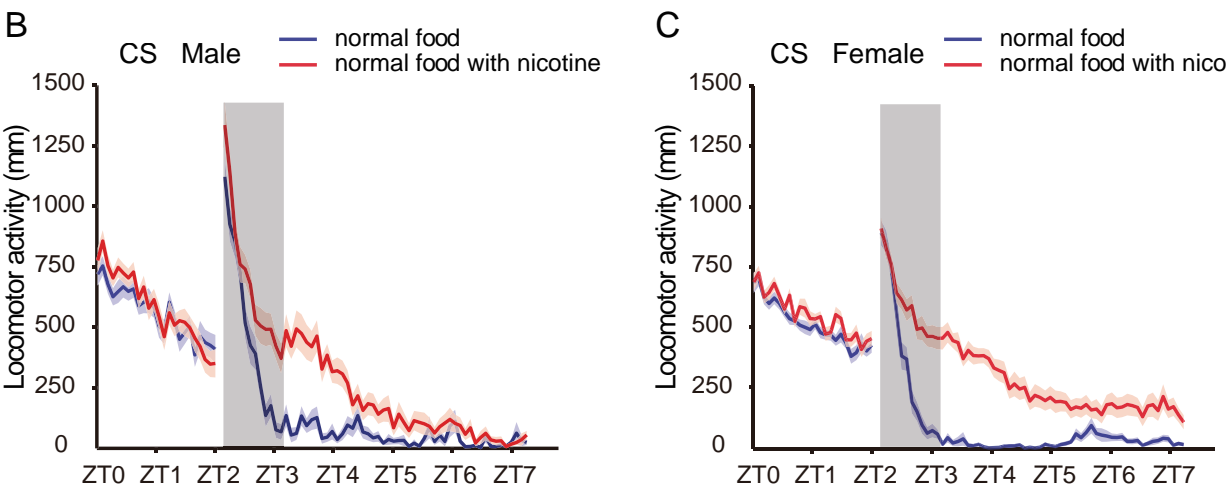

D

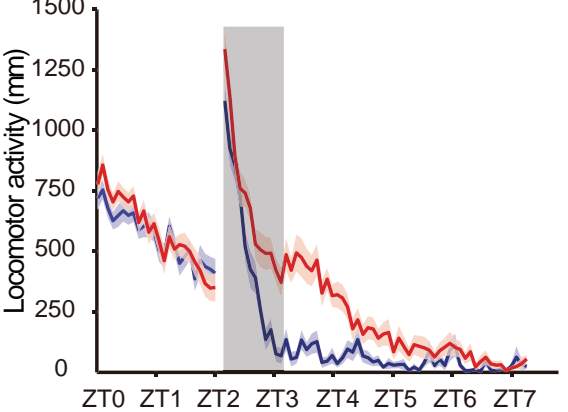

ह
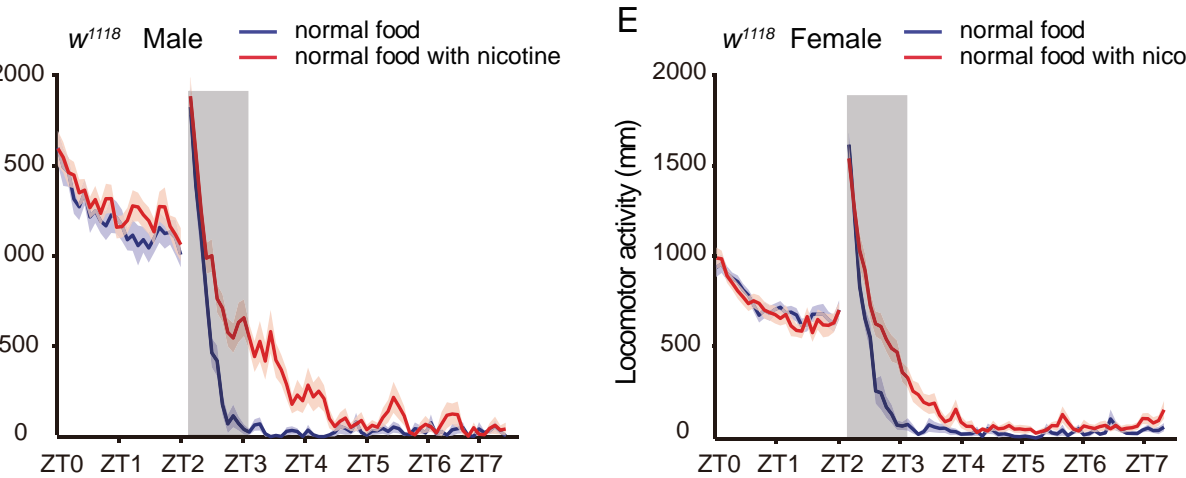


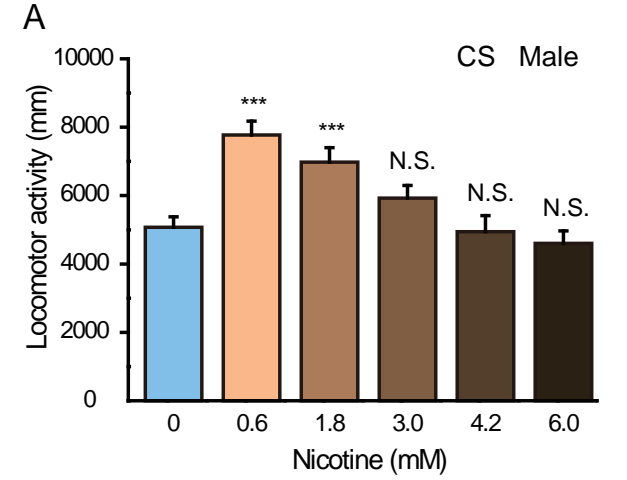

C

B

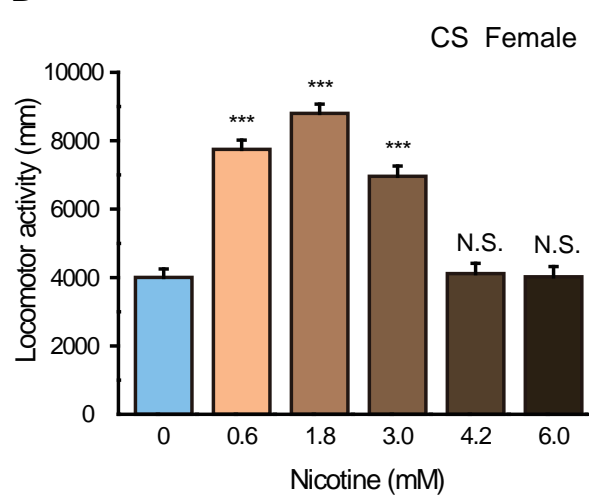

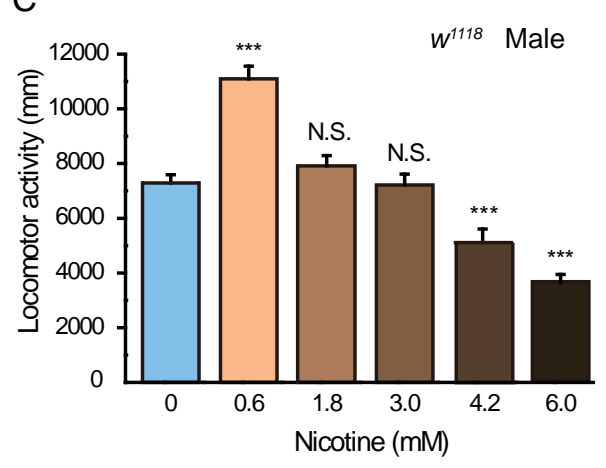

D

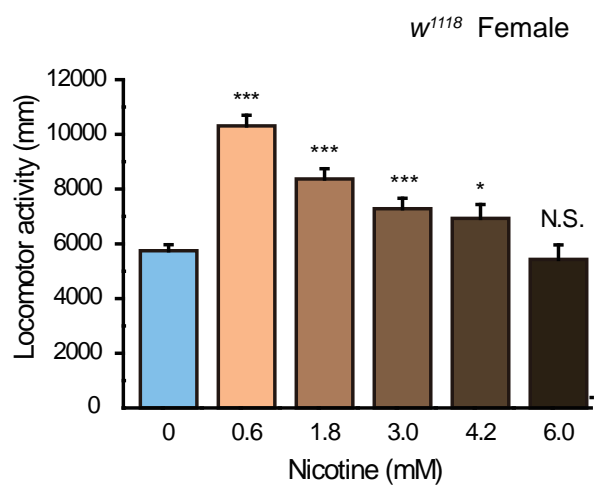

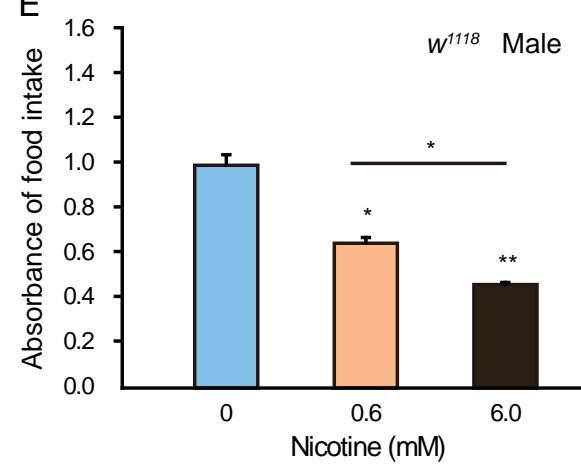

F

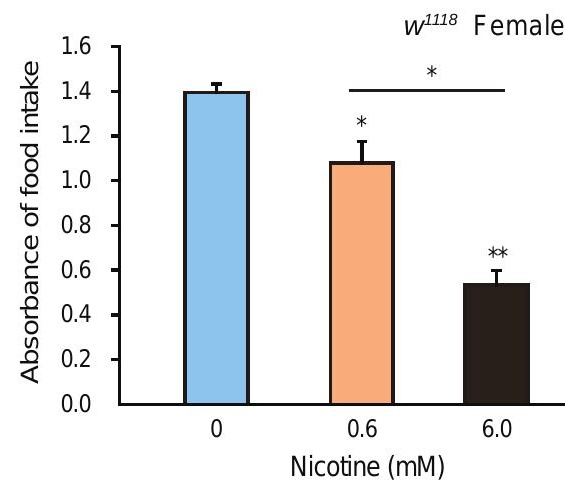


A

$30^{\circ} \mathrm{C}$

$25^{\circ} \mathrm{C}$

ZT12

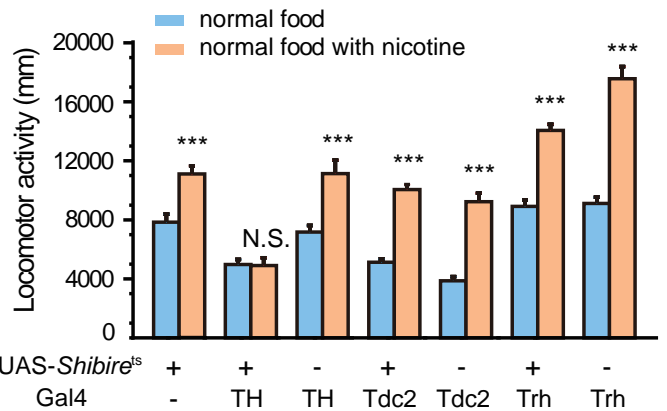

C

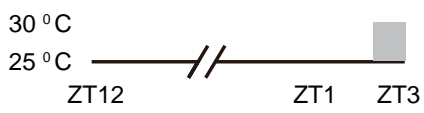

Male

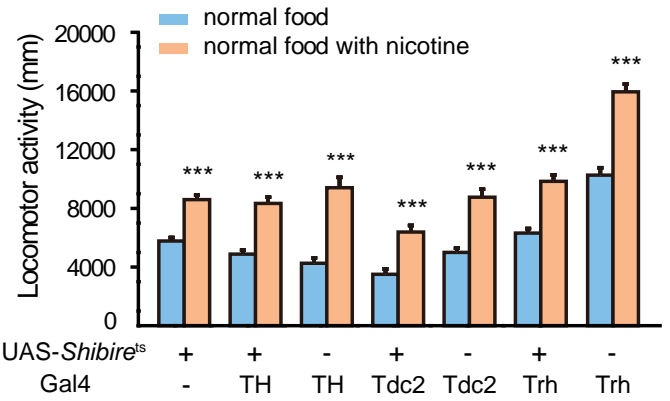

B ZT1 ZT1 ZT3

Female

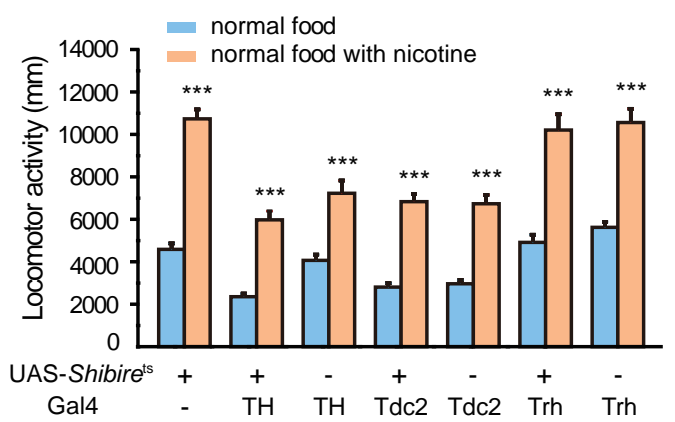

D
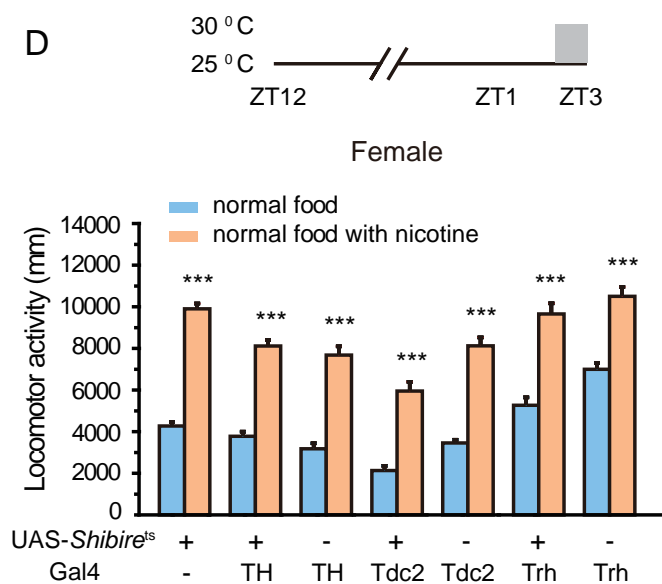
normal food

normal food with nicotine

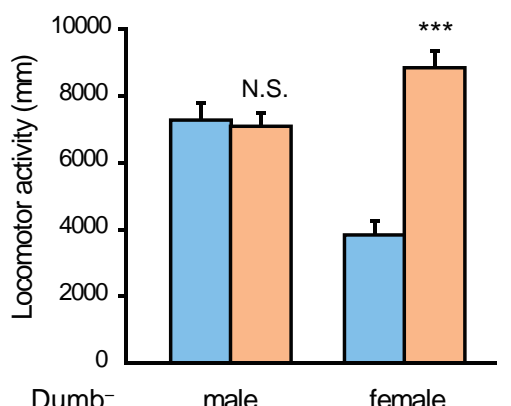

Dumb
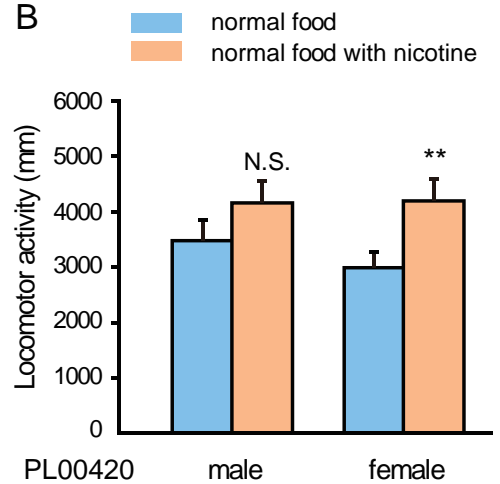

Male

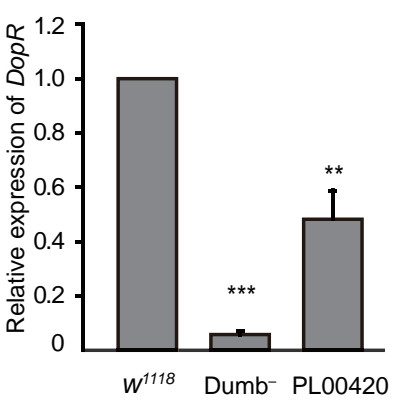

Female

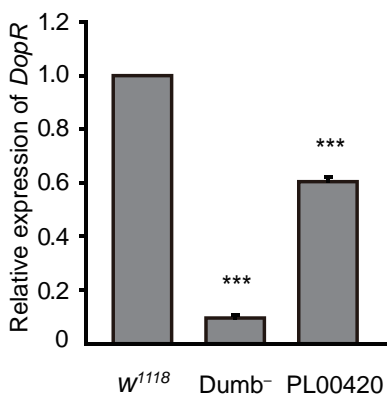

F

Male
E

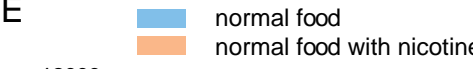

Male

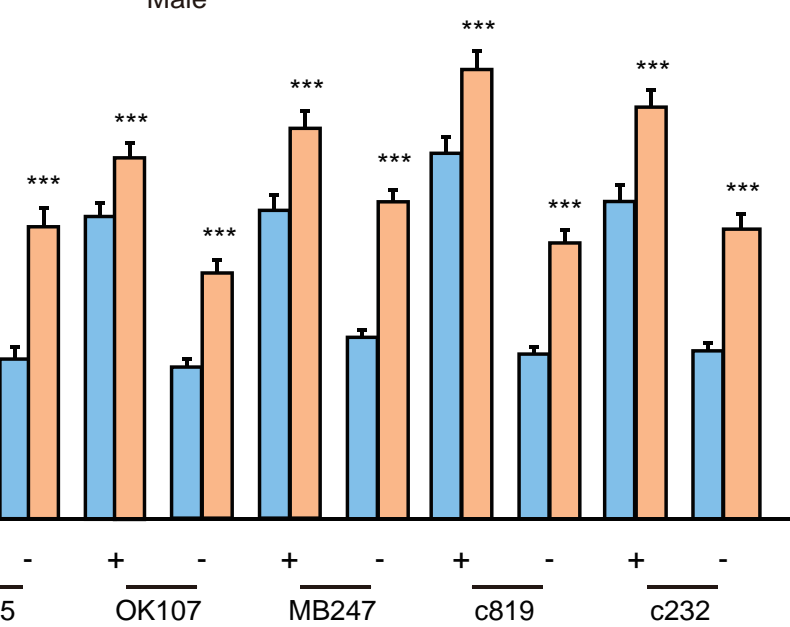

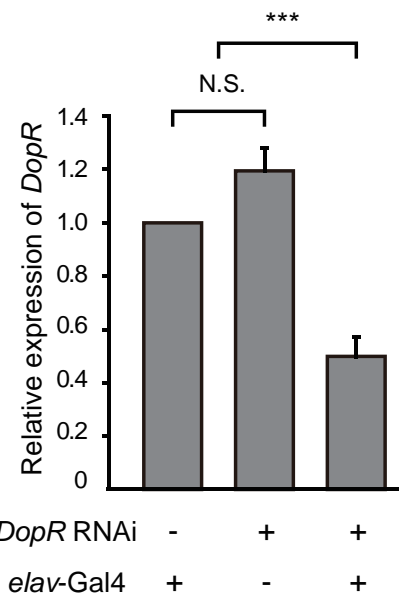




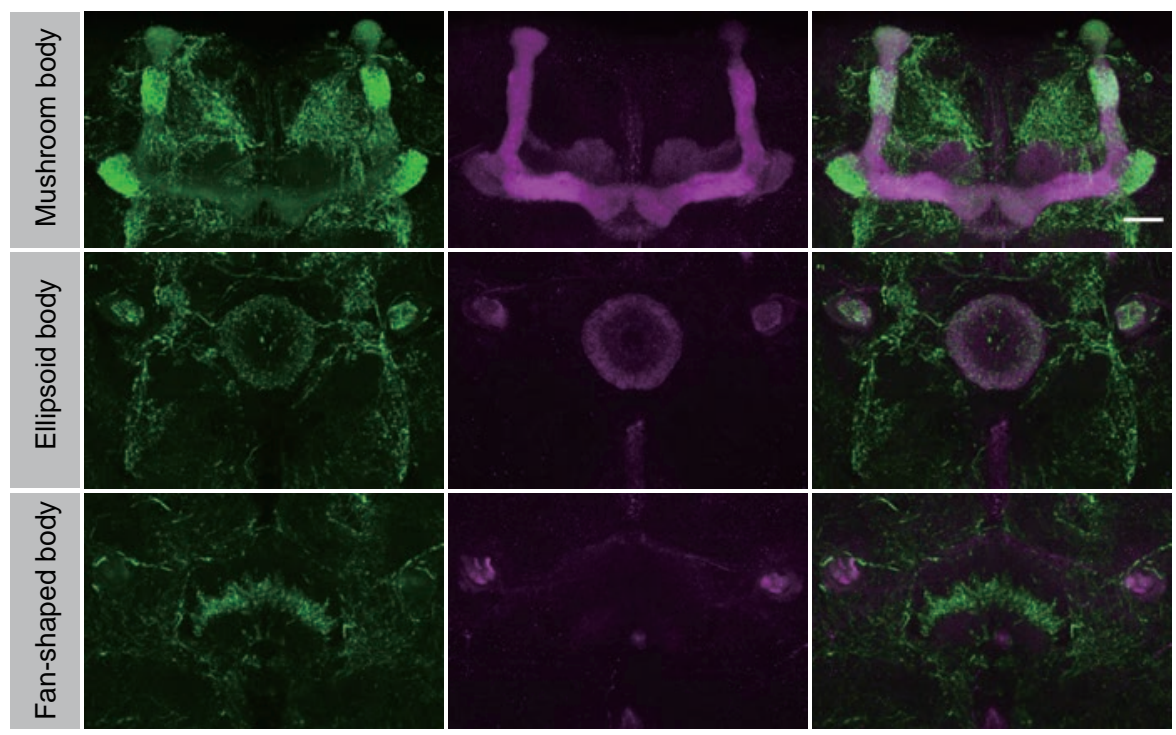

.

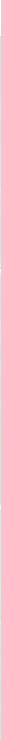


Male

A

Mushroom body

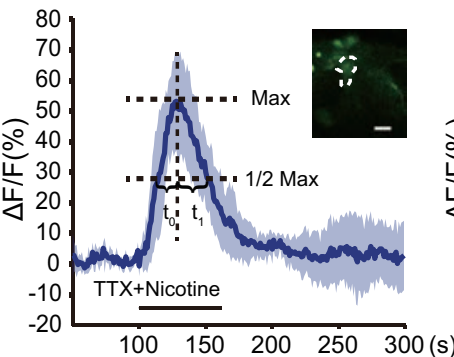

D

Mushroom body

$$
\frac{\widehat{o}}{\frac{1}{4}}
$$

६
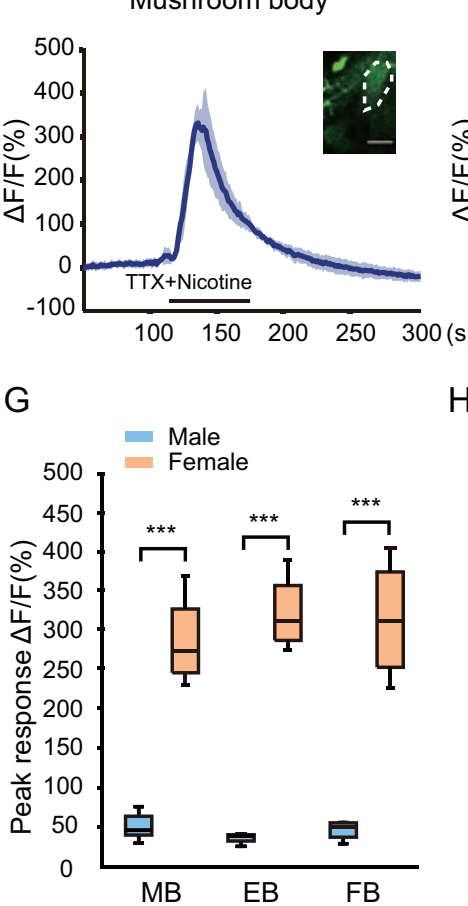

$\mathrm{H}$
B

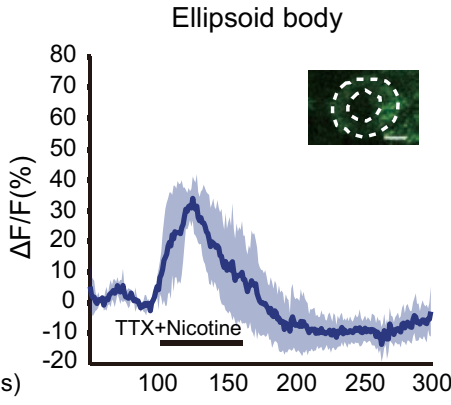

C

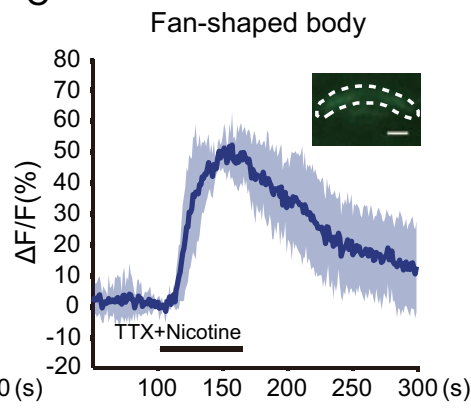

\section{Female}

E

Ellipsoid body

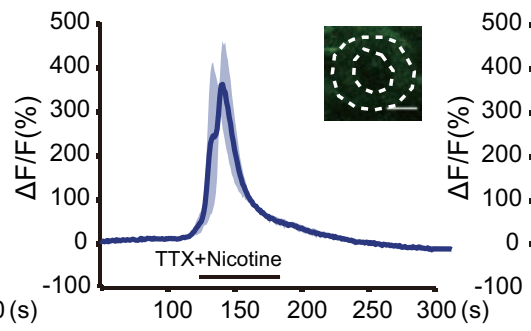

Fan-shaped body

F

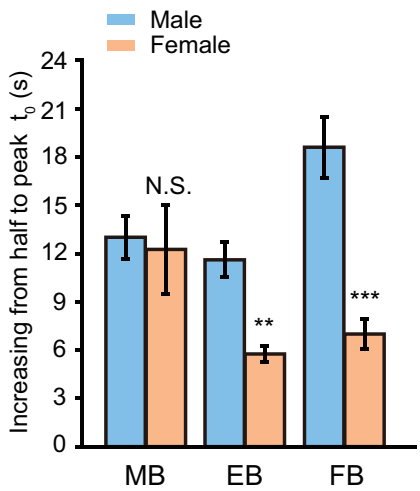

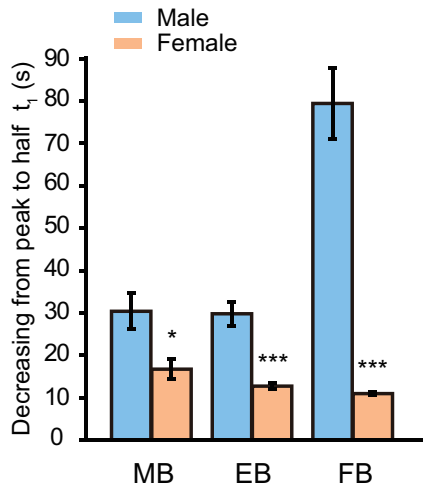


A Male $\quad \begin{aligned} & \text { normal food } \\ & \text { normal food with nicotine }\end{aligned}$

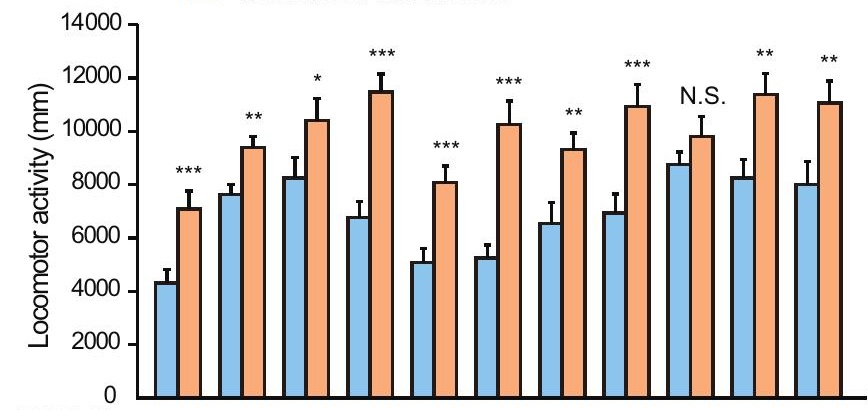

TH-Gal4>

$\begin{array}{llllllllllll}\text { nAchR-RNAi } & - & \alpha 1 & \alpha 2 & \alpha 3 & \alpha 4 & \alpha 5 & \alpha 6 & \alpha 7 & \beta 1 & \beta 2 & \beta 3\end{array}$

B Female $\quad \begin{aligned} & \text { normal food } \\ & \text { normal food with nicotine }\end{aligned}$

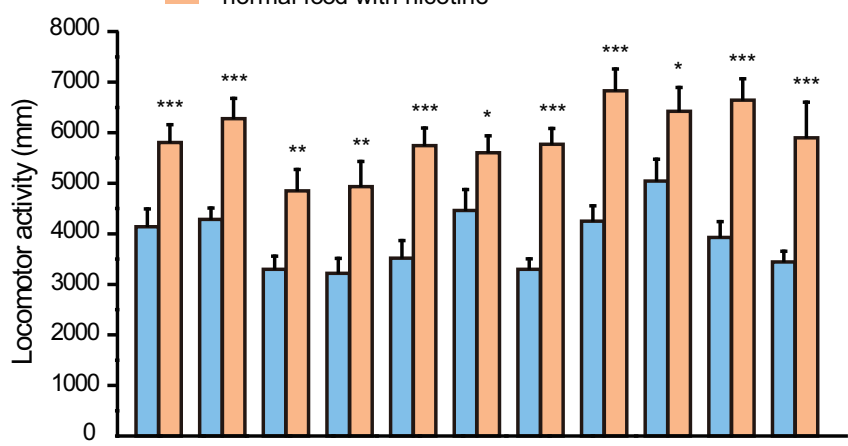

TH-Gal4>

$\begin{array}{llllllllllll}\text { nAchR-RNAi } & - & \alpha 1 & \alpha 2 & \alpha 3 & \alpha 4 & \alpha 5 & \alpha 6 & \alpha 7 & \beta 1 & \beta 2 & \beta 3\end{array}$

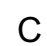

\footnotetext{
normal food

- normal food with nicotine
}

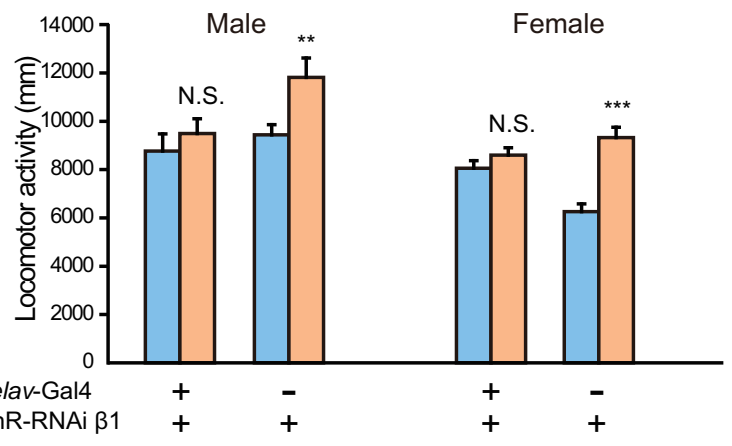

